\title{
Acknowledgement to reviewers
}

Takashi Yagi

\section{Contributing reviewers}

The editors of Genes and Environment would like to thank all our reviewers who have contributed to the journal in Volume XXXVII (2015).

Mie Akanuma
Japan

Yasunobu Aoki

Japan

Michael Fenech

Australia

Kazuo Fujikawa

Japan

Chie Furihata

Japan

Atsushi Hakura

Japan

Shuichi Hamada

Japan

Tsuneo Hashizume

Japan

Yuko Ibuki

Japan

Hiroshi Ide

Japan

Youhei Inaba

Japan

Keiko Inami

Japan

Satoru Itoh

Japan

Nishad Jayasundara

United States of America
Hiroyuki Kamiya
Japan

Kazuaki Kawai

Japan

Makoto Kobayashi

Japan

Seiji Kodama

Japan

Isao Kuraoka

Japan

Yingsong Lin

Japan

Yuji Masuda

Japan

Kenichi Masumura

Japan

Tomonari Matsuda

Japan

Kyomu Matsumoto

Japan

Nan Mei

United States of America

Takeshi Morita

Japan

Yoshimichi Nakatsu

Japan

Takehiko Nohmi

Japan
Shinji Oikawa
Japan

Juraci Alves de Oliveira

Brazil

Angela Risch

Germany

Kazuo Sakai

Japan

Megumi Sasatani

Japan

Kazuhiro Shiizaki

Japan

Yoshiya Shimada

Japan

Kazunori Shiraishi

Japan

Haruhiko Sugimura

Japan

Shizuyo Sutou

Japan

Hitoshi Suzuki

Japan

Takayoshi Suzuki

Japan

Akira Toriba

Japan

Arikuni Uchimura

Japan

Correspondence: yagi-t@riast.osakafu-u.ac.jp

Laboratory of Molecular and Cellular Genetics, Department of Biology,

Graduate School of Science, Osaka Prefecture University, Osaka, Japan 
Keiji Wakabayashi

Japan

Akihiro Wakata

Japan

Masahiko Watanabe Japan
Tetsushi Watanabe

Japan

J Weller

Israel

Masami Yamada

Japan
Ayumi Yamamoto

Japan

Yuichiro Yokota

Japan

Huiping Zhang

United States of America 